\title{
56. DATA REPORT: CARBON ISOTOPE STRATIGRAPHY OF PALEOGENE BULK SEDIMENTS, HOLE 762C (EXMOUTH PLATEAU, EASTERN INDIAN OCEAN) ${ }^{1}$
}

\author{
E. Thomas, ${ }^{2}$ N. J. Shackleton, ${ }^{3}$ and M. A. Hall ${ }^{3}$
}

\begin{abstract}
Carbon isotope measurements were made on bulk sediments from the Paleogene calcareous sequence recovered at Ocean Drilling Program Site 762 (Hole 762C) on the central Exmouth Plateau, eastern Indian Ocean. The very positive $\delta^{13} \mathrm{C}$ values that characterize the early/late Paleocene boundary and the very rapid trend toward lighter values in the latest Paleocene, as observed at other sites worldwide, are clearly present in the record from Hole $762 \mathrm{C}$, as is the short excursion to extremely light values close to the Paleocene/Eocene boundary. The highest values in the upper Paleocene maximum at Site 762 are close to those at mid-latitude South Atlantic sites on Walvis Ridge, but slightly lower than those from the high latitude Sites 689 and 690 (65ㅇ․ Maud Rise, Weddell Sea). These $\delta^{13} \mathrm{C}$ events will be of value in long-distance stratigraphic correlations; especially the short, but extreme excursion at the end of the Paleocene may be useful in clearing up the stratigraphic correlation problems for that interval. Site 762 values for the upper Eocene resemble the pattern at Walvis Ridge more closely than do the values for Sites 689 and 690 (Maud Rise); the latter showed a positive excursion in that interval. The bulk carbon isotopic record seems to be more similar between low- and mid-latitude sites, even in different ocean basins, than between low and high latitudes.
\end{abstract}

\section{INTRODUCTION}

Site $762\left(19^{\circ} 53.23^{\prime} \mathrm{S}, 112^{\circ} 15.24^{\prime} \mathrm{E}\right)$ was drilled in about 1360 $\mathrm{m}$ water on the western flank of the central Exmouth Plateau. Well-preserved Cenozoic nannofossil oozes and chalks were recovered, and recovery with the extended core barrel (XCB) was reasonably good but not excellent $(66.8 \%$ for the Paleogene chalk interval studied). The microfossils studied indicate a low-latitude, open-ocean environment of deposition through the Cenozoic (Haq, von Rad, O'Connell, et al., 1990). Despite the great burial depth of the Paleogene section (Table 1), the sediments appear to have preserved a reliable carbon isotope signal.

For most purposes stable isotope analyses of bulk sediments are of very little value, and one has to analyze monospecific or at least monogeneric assemblages of benthic foraminifers and size-controlled, monospecific samples of planktonic foraminifers (e.g., Berger et al., 1978). It is useful, however, to analyze bulk sediment to obtain appropriate data to contribute to our understanding of the history of the oceanic carbon budget (e.g., Shackleton, 1987). In this report we present a data set from Site 762, at low latitudes in the eastern Indian Ocean, to add to and compare with data sets from low latitudes in the Pacific (Shackleton et al., 1985), the mid-latitudes in the southern Atlantic (Renard et al., 1983; Shackleton and Hall, 1984; Shackleton, 1986), and high latitudes (Shackleton and Hall, 1990).

There are significant spatial variations in $\delta^{13} \mathrm{C}$ in ocean surface waters today (Kroopnick et al., 1977), but the spatial variability in the $\delta^{13} \mathrm{C}$ content of surface sediments is small compared with the range of values observed for the Cenozoic (Shackleton, 1987), which suggests that the bulk sediment record of $\delta^{13} \mathrm{C}$ may be useful in stratigraphic correlations. This

\footnotetext{
1 von Rad, U., Haq, B. U., et al., 1992. Proc. ODP, Sci. Results, 122: College Station, TX (Ocean Drilling Program).

2 University of Cambridge, Department of Earth Sciences, Downing Street, Cambridge CB2 3EQ, United Kingdom.

${ }^{3}$ University of Cambridge, Subdepartment of Quaternary Research, Godwin Laboratory, Free School Lane, Cambridge CB2 3RS, United Kingdom.
}

additional data set can help evaluate the stratigraphic use of bulk $\delta^{13} \mathrm{C}$ data.

\section{ANALYTICAL DATA AND CONCEPTS}

Samples from Hole $762 \mathrm{C}$, weighing a few milligrams, were taken from Cores $122-762 \mathrm{C}-2 \mathrm{X}$ through $-41 \mathrm{X}$ (171 to $540 \mathrm{mbsf}$, corresponding to about 35-65 Ma), then dried and vacuum roasted at $400^{\circ} \mathrm{C}$ to remove any organic contaminants. The samples were then reacted with $100 \%$ phosphoric acid at $90^{\circ} \mathrm{C}$ using a VG Isotech Isocarb common acid bath system. The evolved carbon dioxide was analyzed in a VG Isotech SIRA series II mass spectrometer. The results were calibrated to PDB by repeated analysis of a carbonate standard. Analytical accuracy is better than $0.08 \%$.

Measurements are listed in Table 1. As an aid to preliminary evaluation of the data and comparison with records from other sites, each sample is assigned an age based on the magnetostratigraphic record in Galbrun (this volume, chapter 42) and the calcareous nannofossil record in Siesser and Bralower (this volume). The numerical ages follow Berggren et al. (1985), with the nannofossil zone boundary corrections in Aubry et al. (1988). The age tie-points are listed in Table 2.

\section{DISCUSSION}

Figure 1 shows the $8^{13} \mathrm{C}$ record for Hole $762 \mathrm{C}$ plotted vs. depth, and on the time scale obtained from Table 2 . The pronounced upper Paleocene peak in $\delta^{13} \mathrm{C}$ values around 60 $\mathrm{Ma}$ (time scale of Berggren et al., 1985) is clearly present, as is the rapid decline in values at around the Paleocene/Eocene boundary. The peak values of $\delta^{13} \mathrm{C}$ at around $60 \mathrm{Ma}$ are very similar to those at mid-latitude Deep Sea Drilling Project (DSDP) Sites 525, 527, and 528 (Walvis Ridge; Shackleton and Hall, 1984), but lower than those at high-latitude Sites 689 and 690 (Shackleton and Hall, 1990). It has been demonstrated for data from the Maud Rise on planktonic and benthic foraminifers (Stott et al., 1990; Kennett and Stott, 1990, 1991), as well as in bulk values (Shackleton and Hall, 1990), that there is a short "overshoot" to very light $\delta^{13} \mathrm{C}$ values at the end of this long-term decline in $\delta^{13} \mathrm{C}$ values. A coeval negative excursion was observed in sediments from Site 738 (Antarctic Indian 
Table 1. Stable isotope analyses, Hole $762 \mathrm{C}$.

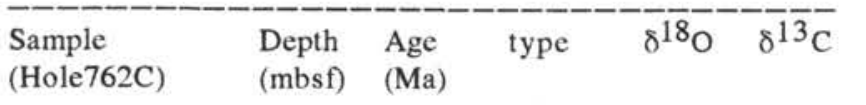

\begin{tabular}{|c|c|c|c|c|c|}
\hline $2 X-1,128-130$ & 171.28 & 35.383 & bulk & 1.09 & 1.83 \\
\hline $2 X-4,85-87$ & 175.35 & 35.576 & bulk & 0.67 & 1.94 \\
\hline $3 \mathrm{X}-1,23-25$ & 179.73 & 35.784 & bulk & 0.63 & 2.25 \\
\hline $3 X-4,72-74$ & 184.72 & 36.021 & bulk & & 2.01 \\
\hline $4 X-1,27-29$ & 189.27 & & bulk & 0.07 & 1.73 \\
\hline $4 X-4,72-74$ & 194.22 & 36.471 & bulk & 0.08 & 1.30 \\
\hline $5 X-1,128-130$ & 199.78 & 36.735 & bulk & -0.12 & 1.50 \\
\hline $6 X-1,132-134$ & 209.32 & 37.187 & bulk & 0.14 & 1.78 \\
\hline $6 X-4,77-79$ & 213.27 & 37.374 & bulk & 0.06 & 1.85 \\
\hline $7 X-1,22-24$ & 217.72 & 37.585 & bulk & -0.19 & 1.72 \\
\hline $7 X-4,72-74$ & 222.72 & 37.830 & bulk & -0.59 & 1.77 \\
\hline $8 X-1,48-50$ & 227.48 & 38.138 & bulk & & 1.89 \\
\hline $8 X-4,71-73$ & & & bulk & & 1.62 \\
\hline $9 X-1,73-75$ & $23^{\prime}$ & 38.7 & bulk & -0 & 1.63 \\
\hline $10 X-1,79-81$ & 246.79 & 39.386 & bulk & 0.10 & 1.81 \\
\hline $10 X-4,72-74$ & 251.22 & 39.898 & bulk & 0.31 & 1.86 \\
\hline $11 X-1,132-134$ & 256.82 & 40.830 & bulk & & \\
\hline $11 X-4,71-73$ & 260.71 & 41.478 & bulk & -0.05 & 2.41 \\
\hline $12 X-1,131-133$ & 266.31 & 42.410 & bulk & 0.28 & 1.87 \\
\hline $12 X-4,72-74$ & 270.22 & 43.061 & bulk & & 1.97 \\
\hline $13 X-1,129-131$ & & & ulk & & 88 \\
\hline $14 \mathrm{X}-1$, & 28 & & bulk & & 1.88 \\
\hline $14 X-4,70-72$ & 289.20 & 46.131 & bulk & 0. & 1.86 \\
\hline $15 X-1,123-125$ & 294.73 & 46.740 & bulk & 0.3 & 1.77 \\
\hline $15 \mathrm{X}-\mathrm{CC}, 13-15$ & 297.50 & 47.0 & bulk & 0. & 1.54 \\
\hline $16 \mathrm{X}-1,121-123$ & 304.21 & 47.765 & bulk & 0.36 & 1.81 \\
\hline $16 \mathrm{X}-3,79-81$ & 306.79 & 48.043 & bulk & 0.0 & 1.90 \\
\hline $17 X-2,32-34$ & 314.32 & 48.857 & bulk & 0. & 1.79 \\
\hline $17 X-5,13$ & 319.81 & 49.450 & bulk & & 70 \\
\hline $18 \mathrm{X}-1,7$ & 32 & 49.716 & bulk & & 1.61 \\
\hline $18 X-2,7$ & 324.29 & 49.934 & bulk & & 1.83 \\
\hline $18 X-3,79-81$ & 325.79 & 50.096 & bulk & & 1.85 \\
\hline $18 X-4,71-73$ & 327.21 & 50.249 & bulk & -0 . & 1.92 \\
\hline $18 X-5,80-82$ & 328.80 & 50.445 & bulk & -0. & 1.32 \\
\hline $18 X-6,79-81$ & 330.29 & 50.654 & bulk & -0.51 & 1.80 \\
\hline $19 X-1,82-84$ & 332.32 & 50.939 & bulk & -0.61 & 1.77 \\
\hline $19 X-2,71-73$ & 333.71 & 51.134 & bulk & & 1.79 \\
\hline $19 X-4,72-74$ & 336.72 & 51.556 & bulk & & 1.48 \\
\hline $20 X-1,82-84$ & 341.82 & 52.074 & bulk & -0 . & 0.77 \\
\hline $20 X-2,71-73$ & 343.21 & 52.149 & & -1.23 & 1.63 \\
\hline $20 X-3,78-80$ & 344.78 & 52.234 & bulk & -1.03 & 0.73 \\
\hline $20 X-4,51-53$ & 346.01 & 52.301 & bulk & -0. & 1.14 \\
\hline $21 X-1,78-80$ & 351.28 & 52.586 & bulk & -0.5 & 1.07 \\
\hline $21 X-2,67-69$ & 352.67 & 52.705 & & -0.8 & 1.16 \\
\hline $22 X-1,72-74$ & 360.72 & 53.598 & & -0 . & 1.12 \\
\hline $22 X-2,71-73$ & 362.21 & 53.763 & & -0.7 & 1.24 \\
\hline $22 X-3,6$ & 363.60 & 53.917 & & & 0.93 \\
\hline $22 X-4,66-68$ & 365.16 & 54.090 & & -0.71 & 1.11 \\
\hline $22 X-5,67-69$ & 366.67 & 54.258 & bulk & -1.15 & 0.83 \\
\hline $22 X-6,72-74$ & 368.22 & 54.430 & bulk & -1.08 & 1.07 \\
\hline $23 X-1,67-69$ & 370.17 & 54.663 & bulk & -0.90 & 1.04 \\
\hline $23 X-2,66-68$ & 371.66 & 54.811 & bulk & -1.03 & 0.99 \\
\hline $23 X-3,54-56$ & 373.04 & 54.964 & bulk & -1.01 & 0.76 \\
\hline $24 X-1,57-59$ & 379.57 & 55.593 & & -1.03 & 0.96 \\
\hline $24 \mathrm{X}-\mathrm{CC}, 8-10$ & 380.50 & 55.662 & & -1.02 & 1.25 \\
\hline & & 55.957 & & -0.73 & 1.20 \\
\hline $25 X-2,78-80$ & 390.78 & 56.011 & bulk & -0.90 & 1.46 \\
\hline
\end{tabular}

Table 1 (continued).

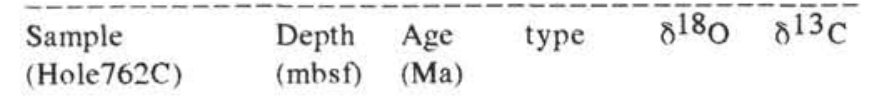

$25 \mathrm{X}-3,70-72$

$25 \mathrm{X}-4,80-82$

$25 \mathrm{X}-5,35-37$

$26 \mathrm{X}-1,15-16$

26X-1, 75- 77

$26 \mathrm{X}-2,15-16$

$26 \mathrm{X}-2,68-70$

$26 \mathrm{X}-3,15-16$

$26 \mathrm{X}-3,67-69$

$26 \mathrm{X}-4,15-16$

$27 \mathrm{X}-1,15-16$

27X-1, 63- 65

$26 \mathrm{X}-4,65-67$

$26 \mathrm{X}-5,15-16$

$27 \mathrm{X}-2,15-16$

27X-2, 61- 63

$26 \mathrm{X}-5,68-70$

$27 \mathrm{X}-3,15-16$

26X-CC, 6- 8

$27 \mathrm{X}-3,65-67$

$27 \mathrm{X}-4,15-16$

$27 \mathrm{X}-4,55-57$

27X-CC,15- 16

$28 \mathrm{X}-1,15-16$

28X-1, 65- 67

28X-CC, $15-16$

28X-CC, 23- 25

$29 \mathrm{X}-1,15-16$

29X-1, 64- 66

$29 \mathrm{X}-2,16-17$

$29 \mathrm{X}-2,65-67$

$29 \mathrm{X}-3,15-16$

$29 \times-3,77-79$

29X-CC, $15-16$

$30 \mathrm{X}-1,15-16$

$30 \mathrm{X}-1,63-65$

$30 \mathrm{X}-2,15-16$

$30 X-2,57-59$

$30 X-3,15-16$

$30 X-3,66-68$

$30 X-4,15-16$

$30 \mathrm{X}-4,57-59$

$30 \mathrm{X}-\mathrm{CC}, 15-16$

$31 \mathrm{X}-1,74-76$

$31 X-2,72-74$

$31 X-3,74-76$

$31 X-4,76-78$

$31 X-5,75-77$

$31 X-6,74-76$

$32 \mathrm{X}-1,75-77$

$32 \mathrm{X}-2,68-70$

32X-CC, 34- 36

$33 \mathrm{X}-1,74-76$

$33 \mathrm{X}-2,71-73$

$33 \mathrm{X}-3,66-68$

$33 \mathrm{X}-4,68-70$

$392.20 \quad 56.059$ bulk

$393.80 \quad 56.114$ bulk

$394.85 \quad 56.164$ bulk

$398.15 \quad 56.437$ fine

$398.75 \quad 56.514$ bulk

$399.65 \quad 56.595$ fine

400.1856 .642 bulk

401.1656 .730 fine

401.6756 .776 bulk

402.6556 .863 fine

402.6656 .864 fine

403.1356 .907 bulk

403.1556 .908 bulk

$404.15 \quad 56.998$ fine

404.1656 .999 fine

404.6157 .039 bulk

404.6857 .046 bulk

$405.66 \quad 57.134$ fine

405.8657 .151 bulk

$406.15 \quad 57.177$ bulk

$407.16 \quad 57.267$ fine

$407.55 \quad 57.303$ bulk

$408.79 \quad 57.414$ fine

412.1657 .716 fine

$412.65 \quad 57.760$ bulk

$413.88 \quad 57.868$ fine

$413.95 \quad 57.877$ bulk

$421.65 \quad 58.567$ fine

422.1458 .611 bulk

$423.16 \quad 58.848$ fine

423.6558 .999 bulk

$\begin{array}{ll}424.65 & 59.249 \\ 4 & \text { fine }\end{array}$

425.2759 .275 bulk

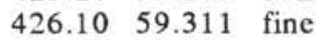

$431.15 \quad 59.527$ fine

431.6359 .547 bulk

$432.65 \quad 59.510$ fine

433.0759 .609 bulk

$434.15 \quad 59.655$ fine

434.6659 .677 bulk

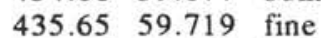

436.0759 .737 bulk

436.6759 .763 fine

441.2459 .959 bulk

442.7260 .022 bulk

444.2460 .087 bulk

$445.76 \quad 60.152$ bulk

447.2560 .216 bulk

$448.74 \quad 60.280$ bulk

450.7560 .366 bulk

$452.18 \quad 60.427$ bulk

453.7560 .494 bulk

460.2460 .772 bulk

461.7160 .835 bulk

$463.16 \quad 60.897$ bulk

$464.68 \quad 60.962$ bulk

466.2061 .027 bulk

\begin{tabular}{|c|c|}
\hline-0.78 & 1.53 \\
\hline-0.92 & 1.48 \\
\hline-0.78 & 0.64 \\
\hline-0.88 & 1.26 \\
\hline-0.85 & 1.15 \\
\hline-1.13 & 1.22 \\
\hline-0.84 & 1.16 \\
\hline-0.95 & 1.26 \\
\hline-0.81 & 1.40 \\
\hline-0.52 & 1.47 \\
\hline-0.89 & 1.30 \\
\hline-0.93 & 1.25 \\
\hline-0.81 & 1.37 \\
\hline-0.55 & 1.37 \\
\hline-0.82 & 1.33 \\
\hline-0.78 & 1.51 \\
\hline-0.79 & 1.34 \\
\hline-0.89 & 1.69 \\
\hline-0.88 & 1.41 \\
\hline-1.04 & 1.41 \\
\hline-1.01 & 1.73 \\
\hline-0.87 & 1.49 \\
\hline-1.09 & 1.84 \\
\hline-0.92 & 1.80 \\
\hline-1.20 & $1.02 *$ \\
\hline-1.28 & 1.29 \\
\hline-0.81 & 2.33 \\
\hline-0.94 & 2.67 \\
\hline-1.03 & 2.05 \\
\hline-0.92 & 3.43 \\
\hline-0.76 & 2.81 \\
\hline-0.83 & 3.07 \\
\hline-0.68 & 2.80 \\
\hline-0.83 & 3.28 \\
\hline-0.74 & 2.99 \\
\hline-0.47 & 3.11 \\
\hline-0.63 & 3.19 \\
\hline-0.51 & 3.01 \\
\hline-0.65 & 3.28 \\
\hline-0.35 & 2.94 \\
\hline-0.61 & 3.15 \\
\hline-0.49 & 2.88 \\
\hline-0.45 & 3.23 \\
\hline-0.69 & 2.56 \\
\hline-0.07 & 3.11 \\
\hline-0.33 & 3.11 \\
\hline-0.61 & 3.06 \\
\hline-0.55 & 3.07 \\
\hline-0.46 & 2.67 \\
\hline-0.44 & 2.69 \\
\hline-0.25 & 2.80 \\
\hline-0.59 & 2.42 \\
\hline-0.70 & 2.72 \\
\hline-0.52 & 2.45 \\
\hline-0.80 & 2.60 \\
\hline-0.75 & 2.39 \\
\hline-0.61 & 2.18 \\
\hline
\end{tabular}

Note: *End Paleocene benthic foraminiferal extinction 
Table 1 (continued).

\begin{tabular}{|c|c|c|c|c|c|}
\hline $\begin{array}{l}\text { Sample } \\
\text { (Hole762C) }\end{array}$ & $\begin{array}{l}\text { Depth } \\
\text { (mbsf) }\end{array}$ & $\begin{array}{l}\text { Age } \\
(\mathrm{Ma})\end{array}$ & type & $\delta^{18} \mathrm{O}$ & $8^{13} \mathrm{C}$ \\
\hline $33 X-6,76-78$ & 467.76 & 61.094 & bulk & -0.62 & 2.40 \\
\hline $34 X-1,73-75$ & 469.73 & 61.178 & bulk & -0.74 & 2.47 \\
\hline $34 X-2,69-71$ & 471.19 & 61.241 & bulk & -0.83 & 2.22 \\
\hline $34 X-3,75-77$ & 472.75 & 61.308 & bulk & -0.60 & 2.08 \\
\hline $34 X-4,79-81$ & 474.29 & 61.374 & bulk & -0.60 & 2.13 \\
\hline $34 X-5,70-72$ & 475.70 & 61.434 & bulk & -0.42 & 2.17 \\
\hline $35 X-1,61-63$ & 479.11 & 61.580 & bulk & -0.73 & 1.93 \\
\hline $35 X-2,63-65$ & 480.63 & 61.645 & bulk & -0.51 & 1.89 \\
\hline $36 \mathrm{X}-\mathrm{CC}, 34-36$ & 488.60 & 61.986 & bulk & -0.74 & 1.67 \\
\hline $37 X-1,78-80$ & 498.28 & 62.401 & bulk & -0.62 & 1.67 \\
\hline $37 X-4,77-79$ & 502.77 & 62.593 & bulk & -0.80 & 1.92 \\
\hline $38 X-1,73-75$ & 507.73 & 62.805 & bulk & -0.56 & 1.91 \\
\hline $38 X-4,76-78$ & 512.26 & 62.999 & bulk & -0.87 & 1.69 \\
\hline $39 X-1,76-78$ & 517.26 & 63.410 & bulk & -0.75 & 1.49 \\
\hline $39 X-4,69-71$ & 521.69 & 64.374 & bulk & -1.10 & 1.29 \\
\hline $40 X-1,73-75$ & 526.73 & 64.587 & bulk & -1.00 & 1.13 \\
\hline $40 X-4,73-75$ & 531.23 & 64.766 & bulk & -0.98 & 1.43 \\
\hline $41 X-1,77-79$ & 536.27 & 64.956 & bulk & -1.11 & 1.34 \\
\hline $41 X-4,73-75$ & 540.73 & 65.146 & bulk & -1.07 & 1.44 \\
\hline
\end{tabular}

Note: Age estimates after Galbrun (this volume, chapter 42) and Siesser and Bralower (this volume); Table 2.

Table 2. Age tie-points used to determine the ages of samples, as shown in Table 1.

\begin{tabular}{lll}
\hline Datum & $\begin{array}{l}\text { Depth, } \\
\text { mbsf }\end{array}$ & $\begin{array}{l}\text { Age, } \\
\text { Ma }\end{array}$ \\
\hline NP20/NP21 & 199.05 & 36.7 \\
NP20/NP19 & 222.25 & 37.8 \\
C16R/C17N & 249.01 & 39.53 \\
C19N/C19R & 273.46 & 43.60 \\
C19R/C20N & 276.01 & 44.06 \\
C20N/C20R & 289.45 & 46.17 \\
C20R/C21N & 310.22 & 48.75 \\
C21N/C21R & 328.05 & 50.34 \\
C21R/C22N & 339.53 & 51.95 \\
C22N/C22R & 351.90 & 52.62 \\
C24N-1/C24R-1 & 376.70 & 55.37 \\
C24R-1/C24N-2 & 380.44 & 55.66 \\
C24N-2/C24R-2 & 394.58 & 56.14 \\
C24R-2/C25N & 422.46 & 58.64 \\
C25N/C25R & 424.45 & 59.24 \\
C26R/C27N & 512.98 & 63.03 \\
C27N/C27R & 518.73 & 63.54 \\
C27R/C28N & 519.59 & 64.29 \\
C28R/C29N & 540.39 & 66.50
\end{tabular}

Ocean; Barrera and Keller, 1991) and from the Walvis Ridge (Thomas and Shackleton, unpubl. data). This short negative peak occurs at the same level as the extinction of many species of deep-sea benthic foraminifers, including Gavelinella beccariiformis (Thomas, 1990; Kennett and Stott, 1991). At Site 762 there is a similar but less extreme drop in $\delta^{13} \mathrm{C}$ values of bulk carbonate at the extinction of $G$. beccariiformis (Fig. 2). This short-lived event could thus be a valuable tool for high-precision stratigraphic correlation in the interval around the Paleocene/Eocene boundary, where stratigraphy is extremely difficult because of the brackish character of the type sections of the Paleocene (Aubry et al., 1986, 1988).

At high-latitude Sites 689 and 690 there is an interval of highly positive $\delta^{13} \mathrm{C}$ values in the uppermost Eocene (around 37-39 Ma). This interval is not present at Site 762 and is likewise absent at the mid-latitude Walvis Ridge sites (Shackleton and Hall, 1984; 1990). Thus it appears that the bulk carbon isotope record can be very useful in some parts of the geological record, but it needs to be interpreted with care.

\section{ACKNOWLEDGMENTS}

We are grateful to Dr. S. O'Connell, the ODP shipboard scientist for Leg 122, for help in obtaining samples, in getting this report presented in Volume 122, and in assisting in obtaining the information needed to document the age-model for Site 762. We thank one anonymous reviewer for comments on the manuscript. This is University of Cambridge DES contribution no. 2069.

\section{REFERENCES}

Aubry, M.-P., Berggren, W. A., Kent, D. V., Flynn, J. J., Klitgord, K. D., Obradovich, J. D., and Prothero, D. R., 1988. Paleogene geochronology: an integrated approach. Paleoceanography, 3:707-742.

Aubry, M.-P., Hailwood, E., and Townsend, 1986. Magnetic and calcareous nannofossil stratigraphy of the lower Palaeogene formations of the Hampshire and London Basins. J. Geol. Soc. 143:729-735.

Barrera, E., and Keller, G., 1991. Late Paleocene to early Eocene climatic and oceanographic events in the Antarctic Indian Ocean. GSA Progr. Abstr.,

Berger, W. H., Killingley, J. S., and Vincent, E., 1978. Stable isotopes in deep-sea carbonates: Box-Core ERDC-92, West Equatorial Pacific. Oceanologica Acta 1:203-216.

Berggren, W. A., Kent, D. V., Flynn, J. J., and Van Couvering, J. A., 1985. Cenozoic geochronology. Geol. Soc. Am. Bull., 96:14071418.

Haq, B. U., von Rad, U., O'Connell, S., et al., 1990. Proc. ODP, Init. Repts., 122: College Station, TX (Ocean Drilling Program)

Kennett, J. P., and Stott, L. D., 1990. Proteus and Proto-Oceanus: ancestral Paleogene oceans as revealed from Antarctic stable isotopic results. In Barker, P. F., Kennett, J. P., et al., Proc. ODP, Sci. Results, 113: College Station, TX (Ocean Drilling Program), 865-880

Kennett, J. P., and Stott, L. D., 1991. Terminal Paleocene deep-sea benthic crisis: sharp deep-sea warming and paleoceanographic changes in Antarctica. Nature,

Kroopnick, P. M., Margolis, S. V., and Wong, C. S., 1977. $\delta^{13} \mathrm{C}$ variations in marine carbonate sediments as indicators of the $\mathrm{CO}_{2}$ balance between the atmosphere and oceans. In Andersen, N. R., and Malahoff, A. (Eds.), The Fate of Fossil Fuel $\mathrm{CO}_{2}$ in the Oceans: New York (Plenum), 295-321.

Renard, M., Richebois, G., and Letolle, R., 1983. Trace element and stable isotope geochemistry of Paleocene to Coniacian carbonate samples from Hole $516 \mathrm{~F}$, comparison with North Atlantic and Tethys sites. In Barker, P. F., Carlson, Johnson, D. A., et al., Init. Repts. DSDP, 72: Washington (U.S. Govt. Printing Office), 399420.

Shackleton, N. J., 1986. Palaeogene stable isotope events. Palaeogeogr., Palaeoclimatol., Palaeoecol., 57:91-102.

Shackleton, N. J., 1987. Carbon isotope record of the Cenozoic. In Brooks, J., and Fleet, A. J. (Eds.), Marine Petroleum Source Rocks: Oxford (Blackwell), 427-438.

Shackleton, N. J., and Hall, M. A., 1984. Carbon isotope data from Leg 74 sediments. In Moore, T. C., Rabinowitz, P. D., et al., Init. Repts. DSDP, 74: Washington (U.S. Govt. Printing Office), 613-619.

Shackleton, N. J., and Hall, M. A., 1990. Carbon isotope stratigraphy of bulk sediments, ODP Sites 689 and 690, Maud Rise, Antarctica. In Barker, P. F., Kennett, J. P., et al., Proc. ODP. Sci. Results, 113: College Station, TX (Ocean Drilling Program), 985-989. 
Shackleton, N. J., Hall, M. A., and Bleil, U., 1985. Carbon isotope stratigraphy, Site 577. In Heath, G. R., Burckle, L. D., et al., Init. Repts. DSDP, 86: Washington (U.S. Govt. Printing Office), 503511.

Stott, L. D., Kennett, J. P., Shackleton, N. J., and Corfield, R. M., 1990. The evolution of Antarctic surface waters during the Paleogene: inferences from the stable isotopic composition of planktonic foraminifers, ODP Leg 113. In Barker, P. F., Kennett, J. P. et al., Proc. ODP, Sci. Results, 113: College Station, TX (Ocean Drilling Program), 849-864
Thomas, E., 1990. Late Cretaceous through Neogene deep-sea benthic foraminifers (Maud Rise, Weddell Sea, Antarctica). In Barker, P. F., Kennett, J. P., et al., Proc. ODP, Sci. Results, 113: College Station, TX (Ocean Drilling Program), 571-594

Date of initial receipt: 19 July 1991

Date of acceptance: 15 August 1991 Ms 122B-195
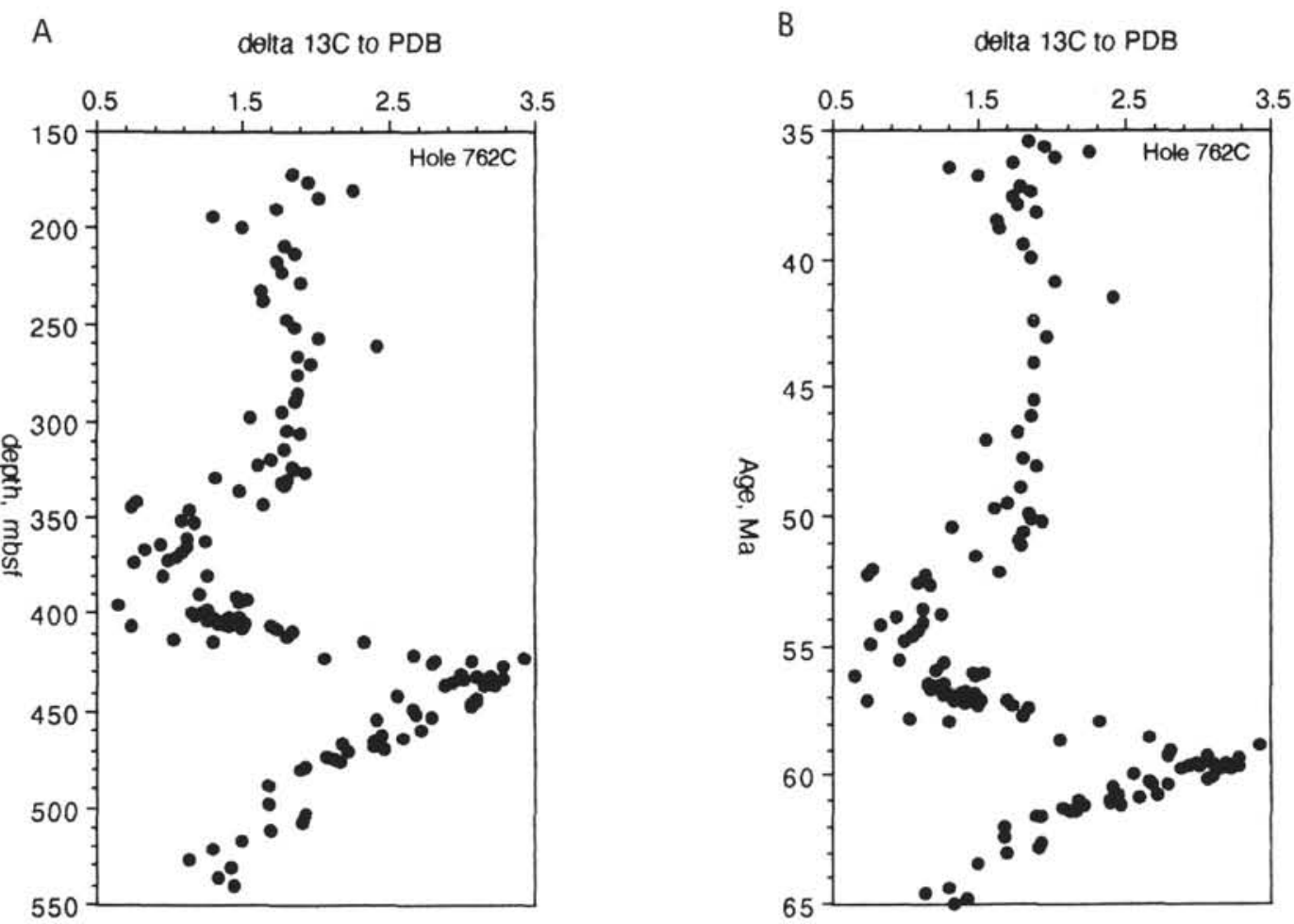

Figure 1. $\delta^{13} \mathrm{C}$ data for bulk sediment in Hole $762 \mathrm{C}$. A. Data plotted against depth in meters below seafloor (mbsf). B. Data plotted against numerical age. Time scale after Siesser and Bralower (this volume) and Galbrun (this volume, chapter 42). 


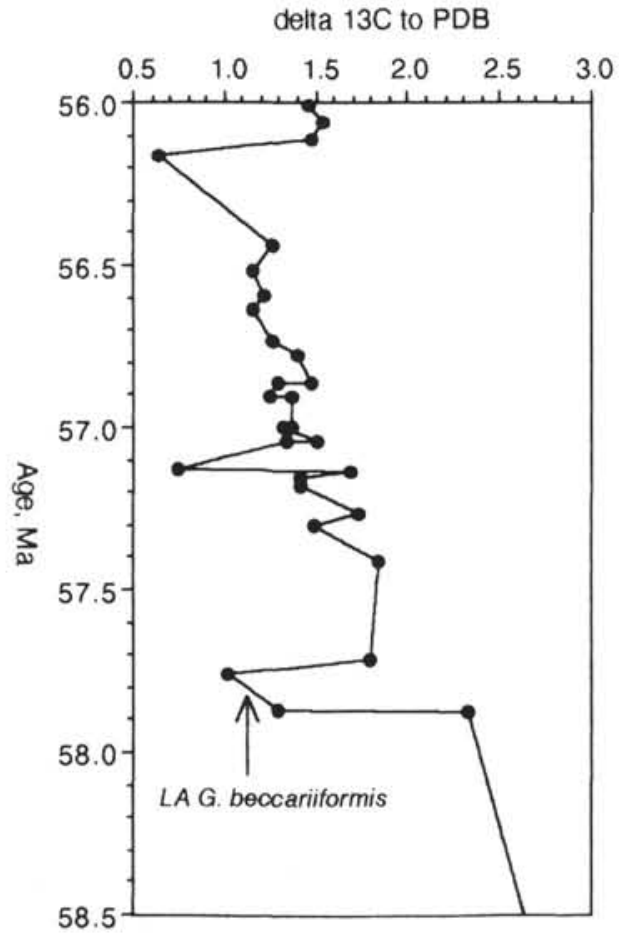

Figure 2. $\delta^{13} \mathrm{C}$ data for bulk sediment in Hole $762 \mathrm{C}$, for the interval between 56.0 and $58.5 \mathrm{Ma}$. The level of last appearance (LA) of Gavelinella beccariiformis is indicated. 